DOI: http://dx.doi.org/10.11144/Javeriana.upsy16-1.eafs

\title{
Efectos del atractivo físico y el sexo en la selección de personal: Un estudio experimental $^{*}$
}

\section{Effects of Sex and Attractiveness in Hiring Decisions: An Experimental Study}

\author{
Ángela Solano-Gómez \\ Universidad de Costa Rica, Costa Rica \\ ORCID: http://orcid.org/0000-0002-2966-2628 \\ Vanessa Smith-Castro \\ Universidad de Costa Rica, Costa Rica
}

\footnotetext{
a Autor de correspondencia. Correo electrónico: angelasolanog@gmail.com
}

Para citar este artículo: Solano-Gómez, A., \& SmithCastro, V. (2017). Efectos del atractivo físico y el sexo en la selección de personal: Un estudio experimental, Universitas Psychologica, 16(1), 1-13. http://dx.doi.org /10.11144/Javeriana.upsy16-1.eafs

\section{RESUMEN}

El presente estudio tuvo como objetivo analizar la activación de información estereotípica en contextos de selección de personal. Las hipótesis fueron contrastadas mediante un diseño experimental 2 (sexo del aplicante) x 2 (atractivo del aplicante) x 2 (sexo de la persona reclutadora) en un grupo de reclutadores profesionales. Los resultados indicaron un efecto significativo tanto del sexo como del atractivo de los estímulos. Estos efectos fueron moderados por el sexo de las personas reclutadoras y parcialmente mediados por las atribuciones de calidez y habilidad. Los resultados se discuten considerando el impacto del estereotipo de "lo bello es bueno" y los estereotipos sobre los roles tradicionales asignados a cada sexo.

\section{Palabras clave}

selección de personal; sexo; atractivo; estereotipos.

\section{ABSTRACT}

This study aimed to analyze the activation of stereotypical information in personnel selection contexts. Hypotheses were tested via a 2 (applicant's sex) $\times 2$ (applicant's appeal) x 2 (sex of the recruiter) experimental design among professional recruiters. Results show a significant effect of both, sex and attractiveness of the stimuli. These effects were moderated by the sex of the recruiter and partially mediated by attributions of warmth and ability. Results are discussed in the light of the impact of the "beautiful is good" effect and the stereotypes of traditional sex roles.

Keywords

personnel selection; sex; attractiveness; stereotypes.

Los procesos de selección de personal llevan implícita la responsabilidad de tomar una decisión racional y objetiva. Sin embargo, las personas reclutadoras no están exentas de presentar sesgos en función de sus experiencias biográficas, los estereotipos culturales, o bien por un mandato explícito de las 
empresas (Ruvalcaba-Coyaso \& Vermonden, 2015; Horcajo, Briñol, \& Becerra, 2009). El presente artículo presenta los resultados de un estudio experimental enfocado en analizar el papel de los estereotipos en contextos de selección de personal.

Dentro de la Psicología Social, el área de investigación en torno a las cogniciones sociales, se ha ocupado del estudio de los estereotipos principalmente en contextos intergrupales, produciendo amplia información sobre los sesgos en la atribución de características de los grupos sociales o etnoculturales y sus implicaciones en las relaciones sociales (Fiske \& Taylor, 2013). Este enfoque de investigación se ha expandido al estudio de los efectos de los estereotipos en las relaciones interpersonales y ha encontrado también aplicación en contextos laborales (Godoy \& Mladinic, 2009).

Dentro de esta última línea de trabajo, numerosos estudios han documentado la existencia de factores cognitivos que afectan la selección de personal, entre las que destacan los estereotipos asociados al sexo y el atractivo físico del aplicante. En el caso del efecto del sexo del aplicante, aunque la evidencia es mixta, un número importante de estudios revelan que los hombres son preferidos en mayor medida que las mujeres en función del tipo de puesto (Caro, García, Rodríguez, \& Jiménez, 2007; Desrumaux, Bosscher, \& Leóni, 2009; Glick, Zion, \& Nelson, 1988; Levin, Rouwenhorst, \& Trisko, 2005; Ng \& Wiesner, 2007; PrzygodzkiLionet, Olivier, \& Desrumaux, 2010; Rice \& Barth, 2016). En lo que respecta al atractivo físico, los resultados también son mixtos; pero existe evidencia considerable de que las personas atractivas tienden a ser privilegiadas en la selección de personal aún en puestos que no requieren de una alta exposición al público (Ndobo, 2014; Sanhueza, Bravo, \& Giusti, 2007; Senior, Thomson, Badger, \& Butler, 2007; Shannon \& Stark, 2003; Sui \& Hong, 2009).

Los intentos de explicación de estos efectos apuntan a diversos factores como inconsistencias entre las habilidades y destrezas de los candidatos y el tipo de puesto (Przygodzki-Lionet et al., 2010) y las preferencias por el mismo sexo
(Gorman, 2005). Sin embargo, parece existir un consenso general alrededor de la idea de que en el proceso de selección se activa información estereotípica que afecta la decisión de reclutamiento (Glick et al., 1988; Godoy \& Mladinic, 2009).

Los estereotipos son creencias socialmente compartidas sobre los atributos y características que poseen las personas, en virtud de su pertenencia a ciertas categorías sociales. Los estereotipos son estructuras cognitivas, subproducto de la evolución de nuestra especie, que permiten a los seres humanos organizar y simplificar el entorno social y con ello comprender el mundo que los rodea (Caprariello, Cuddy, \& Fiske, 2009; Fiske \& Taylor, 2013).

Siendo los estereotipos tan funcionales a nivel cognitivo, parece razonable pensar que estas estructuras cognitivas intervienen también en los procesos de selección de personal. Y en efecto, la investigación ha mostrado que los estereotipos sobre los roles tradicionales de los hombres y las mujeres y los estereotipos asociados al efecto de "lo bello es bueno" parecen tener un papel importante en el contexto de selección de personal (Eagly, Ashmore, Makhijani, \& Longo, 1991; Ndobo, 2014).

Los estereotipos de los roles de hombre y mujer afectan principalmente la selección en puestos tradicionalmente concebidos para uno de los dos sexos. Por ejemplo, la investigación muestra que las mujeres son discriminadas solo cuando el tipo de trabajo es percibido como un trabajo tradicionalmente masculino y se ha observado que los hombres también pueden ser discriminados en ciertos tipos de trabajos (Bauer $\&$ Baltes, 2002). El factor que determina a quién se discrimina parece ser el grado en el que el sexo del aplicante, es congruente o no con la visión estereotípica de las habilidades y destrezas del sexo "apropiado" para el tipo de trabajo (Glick, et al., 1988).

Las hipótesis apuntan a que la naturaleza adaptativa de la división sexual del trabajo, basada en diferentes montos de caza y de recolección para hombres y mujeres (Tooby $\&$ Cosmides, 2005) ha contribuido a una estructuración social de género jerarquizada que 
favorece una categorización social de estímulos masculinos y femeninos en roles sexuales específicos. En el caso particular de la selección de personal, la activación simultánea de roles tradicionales y atributos sexuales parece afectar la selección de los candidatos (Reuben, Sapienza, \& Zingales, 2014).

Los estereotipos de "lo bello es bueno" tienen una influencia en la percepción y evaluación social debido a la asociación del atractivo físico con características positivas. Los estímulos atractivos son más deseables socialmente, se les atribuye mejores habilidades sociales y mayor salud mental y física, se les considera exitosos en las relaciones interpersonales y laborales y se les percibe como más educados, agradables, inteligentes, diligentes, dominantes, felices, cálidos y competentes que los estímulos menos atractivos (Farley, Chia, \& Allred, 1998; Honekopp, Bartholomé, \& Jansen, 2004; Patzer, 2006; Viswesvaran, Schmidt, \& Ones, 2005).

Algunos autores ligan este estereotipo con los mecanismos propios de selección sexual, en la medida en que los rasgos atractivos son una señal de valor como pareja, de más recursos, de cuidados paternales, de un riesgo menor de contagio y de beneficios genéticos como la resistencia a enfermedades (Penton-Voak et al., 2001; Rhodes, 2005).

El presente estudio se concentra en el impacto del atractivo y el sexo de potenciales candidatos a un puesto de trabajo en la decisión de reclutarlos y en las atribuciones estereotípicas de su calidez y habilidad. Se presta especial atención a las atribuciones de calidez y habilidad, porque muchos de los atributos contenidos en los estereotipos de "lo bello es bueno" y de los roles sexuales pueden subsumirse en estas dos dimensiones básicas de atribución. La calidez, implica la atribución de características como cordialidad, simpatía, sinceridad y amistad; mientras que la habilidad hace referencia a la atribución de características como competencia, destreza, seguridad y capacidad. Numerosos estudios han mostrado la capacidad de estas dos dimensiones básicas para sintetizar la mayoría de las adjetivaciones y atributos sociales a nivel intercultural (ver Cuddy et al., 2009). Además, se ha observado que la calidez y la habilidad predicen sentimientos y conductas específicas y representan una conceptualización útil para estudiar las atribuciones que hacemos de las intenciones de las personas y su capacidad de ejecutarlas (Smith-Castro \& Pérez, 2007).

Las hipótesis del estudio predicen que los estímulos masculinos y los estímulos atractivos tenderán a ser favorecidos. Adicionalmente se hipotetiza que las atribuciones de habilidad y calidez median el efecto del atractivo y el sexo sobre la intención de reclutamiento. Se explora además el potencial impacto del sexo de la persona reclutadora en la selección, pero no se adelantan hipótesis sobre esta variable, debido a la poca evidencia inequívoca al respecto.

Datos de la Organización Internacional del Trabajo (OIT) indican que en nuestro contexto todavía persisten desigualdades en la remuneración y en el acceso a las oportunidades de empleo que afectan el desarrollo y realización de las personas (OIT, 2007); de allí la necesidad de promover estudios, como el presente, para fortalecer nuestros conocimientos sobre posibles fuentes de inequidad, incluyendo los estereotipos culturales que se activan en los procesos concretos de selección de personal.

A continuación, se presentan los procedimientos para contrastar las hipótesis propuestas.

\section{Método}

\section{Participantes}

Participaron en el estudio 153 reclutadores, 120 mujeres y 33 hombres del Gran Área Metropolitana de Costa Rica, con edades entre los 20 y 56 años $(M=31.02$ años, $D E=7.61$ años), quienes contaban con una experiencia de 2 meses a 20 años en procesos de selección de personal, con una media de 58.51 meses $(D E=$ 54.15 meses). Los y las participantes pertenecían a un total de 82 empresas de todos los sectores laborales. El 34 \% estudiaron la carrera de Psicología, el 28.1 \% la carrera de Administración 
de Empresas, el 16.3 \% de Recursos Humanos y un $21.6 \%$ otras carreras.

\section{Materiales y estímulos}

Fotografías. Los estímulos eran cuatro Curriculum Vitaes (CVs) cuya única diferencia radicaba en las fotografías de los aspirantes al puesto. Las fotografías se seleccionaron de una base de 50 fotografías de personas costarricenses (50\% de mujeres), quienes participaron voluntariamente como modelos. Las fotografías presentaban personas con edades entre los 22 y los 27 años, de piel clara (blancos/mestizos), que no utilizaban anteojos y no aparecían sonriendo. Todos los modelos utilizaron una camiseta negra lisa. En el caso de los hombres, ninguno tenía barba o bigote y tenían el cabello corto. En el caso de las mujeres aparecían sin maquillaje y sin joyas.

Las fotografías fueron evaluadas por 20 estudiantes de Psicología (50 \% hombres), quienes puntuaron cada fotografía en una escala de 1 "poco atractivo" a 10 "muy atractivo". A partir de esta evaluación se obtuvieron las fotografías que operacionalizaban las condiciones de los factores intrasujetos: un hombre atractivo ( $\mathrm{M}$ en atractivo $=5.85, D E=2.39)$, un hombre menos atractivo $(\mathrm{M}$ en atractivo $=1.80, \mathrm{DE}=1.28)$, una mujer atractiva $(M=5.60, D E=1.43)$, y una mujer menos atractiva $(M=1.55, D E=$ 0.89). Los estímulos atractivos fueron percibidos como significativamente más atractivos que los estímulos menos atractivos (masculinos: $t_{(19)}=$ 6.60, $p<0.001$, femeninos: $t_{(19)}=10.12, p$ $<0.001$ ). Además, se tuvo la certeza de que la fotografía de la mujer atractiva no fuera percibida como significativamente más o menos atractiva que la fotografía del hombre atractivo ( $t$ (19) $=0.42, p=0.683)$ y que la fotografía de la mujer menos atractiva no fuera percibida como significativamente más o menos atractiva que la fotografía del hombre menos atractivo $\left(t_{(19)}=\right.$ $1.16, p=0.262$ ).

El puesto. Para seleccionar un puesto que no fuera estereotípicamente asociado al sexo o el atractivo de las personas, se recurrió a la evaluación por parte de jueces expertos. Dos profesionales en el área de selección de personal (con una experiencia media de 13 años, $D E=$ 10.58 años) evaluaron 21 puestos indicando si el atractivo físico o el sexo tenían una influencia a la hora de realizar una contratación para el mismo. Lo puestos no exigían contacto directo con personas. Los resultados indicaron que el puesto menos asociado al atractivo físico o al sexo fue el de "Agente de Call Center" (operador telefónico) (acuerdo entre jueces Kappa de Cohen $=0.66, p$ $<0.001)$.

Descripción y perfil de puesto. Para presentar una descripción y un perfil del puesto lo más realista posible se trabajó con el acuerdo entre otros dos profesionales con experiencia en selección de personal ( $M=6$ años, $D E=$ 2.83). En la descripción del puesto consideraron aspectos relevantes como las funciones, los requisitos, las competencias y los conocimientos.

\section{Diseño}

Las hipótesis fueron contrastadas mediante un diseño $2 \times 2 \times 2: 2$ sexo (estímulos masculinos vs estímulos femeninos) $\mathrm{x} 2$ atractivo (estímulos atractivos vs menos atractivos), $\mathrm{x} 2$ sexo de la persona reclutadora (hombre vs mujer). Los dos primeros como factores intrasujetos y el último como factor entresujetos.

\section{Procedimiento}

Para la recolección de la información se confeccionaron folletos que incluían: a) el consentimiento informado siguiendo los lineamientos del comité de ética de la Universidad de Costa Rica, b) los cuatro CVs, c) las escalas para medir la Calidez y Habilidad de los postulantes, d) una pregunta sobre la intención de dar el puesto a la persona cuyo CV se acababa de leer y e) un módulo de datos demográficos.

Los CVs. Los CVs estaban compuestos por cuatro secciones: a) la fotografía, b) los datos personales c) la formación académica y d) la experiencia laboral. Los participantes evaluaron cuatro distintos CVs correspondientes a la 
combinación de las condiciones de los dos factores intrasujetos: a) hombre atractivo, b) hombre menos atractivo, c) mujer atractiva y d) mujer menos atractiva. Los CVs eran equivalentes en su contenido, con excepción de la fotografía. El orden de presentación de los CVs fue aleatorizado.

\section{Instrumentos}

Calidez y habilidad percibida. Para medir la calidez y habilidad atribuidas a los estímulos se utilizó la adaptación al castellano realizada por Smith-Castro y Pérez (2007) de las escalas de calidez y habilidad de Fiske, Cuddy, Glick y Xu (2002). La escala de calidez está compuesta por cuatro características: cordialidad, simpatía, sinceridad y calidad. La escala de habilidad está compuesta por otros cuatro atributos: competencia, capacidad, habilidad y seguridad. Los estímulos eran calificados mediante una escala Likert de 7 puntos, de 1 (nada en absoluto) a 7 (completamente). Los índices de calidez y habilidad se construyeron a partir del promedio de las repuestas a los respectivos atributos. Las escalas presentaron Alfas de Cronbach superiores a 0.92 .

Intención de selección. Para medir la intención de reclutamiento se utilizó una única pregunta, en donde se solicitaba al participante indicar si le daría el puesto a la persona del CV que acababa de leer en una escala de 1 "definitivamente no" a 7 "definitivamente sî".

Datos demográficos. Los datos demográficos incluían preguntas sobre edad, sexo, años y/ o meses de experiencia, formación académica y nivel educativo de los reclutadores y las reclutadoras.

\section{Resultados}

Tres análisis de varianza mixtos $2 \times 2 \times 2$ se realizaron para analizar el potencial efecto del sexo y el atractivo de los estímulos (las fotografías) y el sexo de los reclutadores sobre la intención de reclutamiento, la calidez percibida y la habilidad percibida de los estímulos respectivamente. La Tabla 1 presenta las medias y desviaciones estándar utilizadas en todos los análisis. ${ }^{1}$

\section{TABLA 1}

Medias y desviaciones estándar de la intención de reclutamiento, la calidez percibida y la habilidad percibida según el sexo de los estímulos, el atractivo de los estímulos y el sexo de las personas reclutadoras

\begin{tabular}{|c|c|c|c|c|c|c|}
\hline \multirow[b]{3}{*}{ Estímulos } & \multicolumn{6}{|c|}{ Intención de reclutamiento } \\
\hline & \multicolumn{2}{|c|}{ Hombre } & \multicolumn{2}{|c|}{ Mujer } & \multicolumn{2}{|c|}{ Total } \\
\hline & $M$ & $D E$ & $M$ & $D E$ & $M$ & $D E$ \\
\hline Masculinos & 4.36 & 1.44 & 4.28 & 1.35 & 4.30 & 1.39 \\
\hline Femeninos & 4.26 & 1.52 & 3.78 & 1.46 & 3.88 & 1.48 \\
\hline Atractivos & 4.76 & 1.46 & 4.73 & 1.49 & 4.72 & 1.48 \\
\hline Menos atractivos & 4.00 & 1.65 & 3.33 & 1.52 & 3.46 & 1.56 \\
\hline Masculino/atractivo & 4.94 & 1.48 & 4.95 & 1.54 & 4.95 & 1.52 \\
\hline Femenino/atractivo & 4.58 & 1.58 & 4.52 & 1.70 & 4.53 & 1.65 \\
\hline Masculino/menos atractivo & 3.97 & 1.58 & 3.61 & 1.57 & 3.69 & 1.57 \\
\hline \multirow[t]{3}{*}{ Femenino/menos atractivo } & 4.03 & 1.76 & 3.04 & 1.65 & 3.25 & 1.72 \\
\hline & \multicolumn{6}{|c|}{ Habilidad percibida } \\
\hline & \multicolumn{2}{|c|}{ Hombre } & \multicolumn{2}{|c|}{ Mujer } & \multicolumn{2}{|c|}{ Total } \\
\hline Estimulos & $M$ & $D E$ & $M$ & $D E$ & $M$ & $D E$ \\
\hline Masculinos & 4.35 & 1.18 & 4.45 & 1.24 & 4.43 & 1.23 \\
\hline Femeninos & 4.22 & 1.13 & 4.11 & 1.30 & 4.14 & 1.26 \\
\hline Atractivos & 4.50 & 1.23 & 4.71 & 1.30 & 4.66 & 1.28 \\
\hline Menos atractivos & 4.08 & 1.14 & 3.86 & 1.36 & 3.90 & 1.32 \\
\hline Masculino/atractivo & 4.62 & 1.31 & 4.80 & 1.35 & 4.76 & 1.34 \\
\hline Femenino/atractivo & 4.98 & 1.23 & 4.61 & 1.38 & 4.10 & 1.35 \\
\hline Masculino/menos atractivo & 4.39 & 1.24 & 4.11 & 1.38 & 4.56 & 1.35 \\
\hline \multirow[t]{3}{*}{ Femenino/menos atractivo } & 4.05 & 1.14 & 3.61 & 1.47 & 3.71 & 1.41 \\
\hline & \multicolumn{6}{|c|}{ Calidez percibida } \\
\hline & \multicolumn{2}{|c|}{ Hombre } & \multicolumn{2}{|c|}{ Mujer } & \multicolumn{2}{|c|}{ Total } \\
\hline Estímulos & $M$ & $D E$ & $M$ & $D E$ & $M$ & $D E$ \\
\hline Masculinos & 4.02 & 0.94 & 4.12 & 1.13 & 4.10 & 1.09 \\
\hline Femeninos & 3.90 & 1.13 & 3.78 & 1.13 & 3.81 & 1.12 \\
\hline Atractivos & 4.57 & 1.28 & 4.69 & 1.24 & 4.66 & 1.22 \\
\hline Menos atractivos & 3.35 & 1.08 & 3.21 & 1.21 & 3.24 & 1.19 \\
\hline Masculino/atractivo & 4.69 & 1.16 & 4.78 & 1.30 & 4.76 & 1.23 \\
\hline Femenino/atractivo & 4.44 & 1.25 & 4.61 & 1.30 & 4.57 & 1.29 \\
\hline Masculino/menos tractivo & 3.35 & 1.17 & 3.46 & 1.29 & 3.44 & 1.26 \\
\hline Femenino/menos atractivo & 3.36 & 1.78 & 2.96 & 1.30 & 3.04 & 1.28 \\
\hline
\end{tabular}

Nota: Mín. = 1, Máx. =7. Altas puntuaciones indican mayor intención de reclutamiento, mayor calidez percibida y mayor habilidad percibida, respectivamente. $M=$ media, $D E=$ Desviación estándar. Fuente: elaboración propia

Intención de reclutamiento. El primer análisis de varianza indicó un efecto principal significativo del sexo $\left(F_{1,145}=14.677, p<0.001\right.$, $\left.\eta^{2}=0.092\right)$ y del atractivo $\left(F_{1,145}=62.07, p\right.$ $<0.001, \eta^{2}=0.300$ ) de las fotografías sobre la intención de reclutamiento, pero cada uno de estos efectos principales se vio restringido por una interacción de dos vías con el sexo de la persona reclutadora $\left(F_{1,145}=4.44, p=0.037, \eta^{2}=\right.$ 0.030 con el sexo de la fotografía y $F_{1,145}=5.55$, $p=0.020, \eta^{2}=0.037$ con el atractivo de la fotografía). Ninguno de los otros posibles efectos o las restantes interacciones de dos o tres vías resultaron estadísticamente significativas (todas las $\mathrm{Fs}_{1,145}<3,428$, todas las ps $>0.066$ ). 
Para analizar con más detalle las interacciones observadas se realizaron dos análisis de varianza intrasujetos 2 (sexo de la fotografía) x 2 (atractivo de la fotografía) para reclutadores hombres y reclutadoras mujeres por separado.

Para el caso de los hombres, se observó un efecto principal del atractivo de los estímulos fotográficos de los potenciales candidatos $\left(F_{1,30}\right.$ $\left.=11.634, p=0.002, \eta^{2}=0.279\right)$. Ningún otro posible efecto principal o de interacción resultó significativo (todas las $F_{s}<2.35$, todas las ps > 0.136). Específicamente, la intención de reclutamiento fue significativamente mayor para los modelos atractivos $(M=4.76$, IC $95 \%$ [4.25, 5.26]) que para los estímulos no atractivos $(\mathrm{M}=$ 4.00, IC $95 \%[3.40,4.60])$.

Para el caso de las mujeres, se observó un efecto principal tanto del sexo de la fotografía ( $F$ $\left.1,115=36.851, p<0.001, \eta^{2}=0.243\right)$, como del atractivo de la fotografía $\left(F_{1,115}=118.939\right.$, $\left.p<0.001, \eta^{2}=0.508\right)$. Específicamente, la intención de reclutamiento fue mayor ante los estímulos masculinos $(M=4.28$, IC $95 \%$ [4.03, 4.53]) que femeninos $(M=3.78$, IC $95 \%$ [3.51, 4.05]) y mayor para los estímulos atractivos ( $M$ $=4.73$, IC $95 \%[4.45,5.01])$ que para los no atractivos $(M=3.33$, IC $95 \%[3.05,3.61])$.

Calidez atribuida a los estímulos. El segundo análisis de varianza indicó un efecto principal significativo del sexo de la fotografía $\left(F_{1,150}=\right.$ $\left.17.76, p<0.001, \eta^{2}=0.106\right)$ y del atractivo de las fotografías $\left(F_{1,150}=159.72, p<0.001, \eta^{2}\right.$ $=.52$ ) sobre la calidez percibida de los estímulos, pero cada uno de estos efectos principales se vio restringido por una interacción de tres vías con el sexo de la persona reclutadora $\left(F_{1,150}=\right.$ 5.26, $\left.p=0.023, \eta^{2}=0.034\right)$. Ninguno de los otros posibles efectos principales o las restantes interacciones -de dos o tres vías- resultaron estadísticamente significativas (todas las $F_{s_{1,150}}$ $<3.887$, todas las ps $>0.051$ ).

Para analizar con más detalle esta interacción de tres vías se llevaron a cabo dos análisis de varianza intra-sujetos 2 (sexo de la fotografía) x 2 (atractivo de la fotografía) para los reclutadores y las reclutadoras por separado.
Para el caso de los reclutadores, se observó un efecto principal del atractivo de los estímulos fotográficos o potenciales candidatos $\left(F_{1,31}=\right.$ 53, $\left.p<0.001, \eta^{2}=0.633\right)$. Ningún otro posible efecto principal o de interacción resultó significativo (todas las $F_{s}<1.96$, todas las ps $>$ 0.171). Específicamente, los estímulos atractivos fueron percibidos como significativamente más cálidos $(\mathrm{M}=4.57$, IC $95 \%[4.16,4.98])$ que los estímulos no atractivos ( $\mathrm{M}=3.35$, IC $95 \%$ $[2.97,3.74])$. Para el caso de las reclutadoras, se observó un efecto principal del sexo de la fotografía $\left(F_{1,119}=43.32, p<0.001, \eta^{2}=\right.$ $0.267)$ y del atractivo de la fotografía $\left(F_{1,119}=\right.$ 215.173, $p<0.001, \eta^{2}=0.645$ ), así como una interacción significativa entre estos dos factores $\left(F_{1,119}=8.71, p=0.002, \eta^{2}=0.068\right)$.

Para analizar esta interacción se analizaron las comparaciones post hoc (con corrección Bonferroni) de un análisis de varianza simple intrasujetos comparando la combinación de estímulos masculinos y femeninos, atractivos y no atractivos solo para la muestra de reclutadoras, lo cual sería equivalente a un análisis de efectos simples en factores intrasujetos (Keppel \& Wickens, 2004).

El patrón de medias indicó que en muestra de reclutadoras los estímulos atractivos fueron considerados como los más cálidos con una media en calidez percibida de 4.78 (IC $95 \%$ [4.54, 5.00]) para los estímulos masculinos atractivos y una media de 4.61 (IC $95 \%$ [4.37, 4.84]) para estímulos femeninos atractivos. Los estímulos menos atractivos fueron considerados como los menos cálidos con una media de 3.46 (IC 95 $\%$ [3.23, 3.69]) y de 2.96 (IC $95 \%$ [2.73, 3.20]) para estímulos masculinos y femeninos, respectivamente.

Ahora bien, al interior de los estímulos atractivos no se encontraron diferencias significativas según su sexo $(p=0.60)$, pero al interior de los estímulos menos atractivos si encontramos diferencias significativas según su sexo $(p<0.001)$.

Habilidad atribuida a los estímulos. El tercer análisis de varianza indicó un efecto principal significativo del sexo $\left(F_{1,149}=15.28, p<0.001\right.$, 
$\left.\eta^{2}=0.09\right)$ y del atractivo $\left(F_{1,149}=46.55, p\right.$ $<0.001, \eta^{2}=0.24$ ) de las fotografías sobre la habilidad atribuida a los estímulos, pero cada uno de estos efectos principales se vio restringido por una interacción de tres vías con el sexo de la persona reclutadora $\left(F_{1,149}=5.92, p=0.016\right.$, $\left.\eta^{2}=0.038\right)$. Ninguno de los otros posibles efectos principales o las restantes interacciones - de dos o tres vías- resultaron estadísticamente significativas (todas las $\mathrm{Fs}_{1,149}<2.94$, todas las ps $>0.088$ ). Para analizar con más detalle esta interacción de tres vías utilizamos la misma estrategia utilizada anteriormente.

Para el caso de los reclutadores, se observó un efecto principal del atractivo de los estímulos fotográficos $\left(F_{1,32}=12.21, p=0.001, \eta\right.$ $2=0.276)$. Ningún otro posible efecto principal o de interacción resultó significativo (todas las Fs $<2.35$, todas las ps $>0.135$. Específicamente, los estímulos atractivos fueron percibidos significativamente más hábiles $(\mathrm{M}=$ 4.50, IC $95 \%[4.07,4.94])$ que los estímulos no atractivos $(M=4.08$, IC $95 \%[3.66,4.47])$.

Para el caso de las reclutadoras se observó un efecto principal significativo del sexo de la fotografía $\left(F \quad 1,118=33.07, p<0.001, \eta^{2}=\right.$ $0.22)$ y del atractivo de la fotografía $(F 1,118=$ $82.83, p<0.001, \eta^{2}=0.42$ ) sobre la percepción de la habilidad de los estímulos. Y al igual que en el caso anterior, se observó un efecto interactivo significativo de estos factores sobre la habilidad atribuida $\left(F 1,118=9.22, p=0.003, \eta^{2}=0.073\right)$.

El patrón de medias indica que en la muestra de reclutadoras los estímulos atractivos fueron considerados como más hábiles que los estímulos no atractivos, con una media en habilidad percibida de 4.80 (IC $95 \%$ [4.55, 5.04]) para estímulos masculinos atractivos y una media de 4. 61 (IC $95 \%$ [4.36, 4.87]) para estímulos femeninos atractivos. Los estímulos menos atractivos fueron considerados por las reclutadoras como los menos cálidos con una media de 4.11 (IC $95 \%[3.86,4.36]$ ) y de 3.61 (IC $95 \%[3.35,3.88])$ para estímulos masculinos y femeninos, respectivamente.
Las pruebas post hoc indicaron que al interior de los estímulos atractivos no se encontraron diferencias significativas según su sexo $(p$ $=0.117$ ), pero al interior de los estímulos menos atractivos si encontramos diferencias significativas según su sexo $(p<0.001)$.

Análisis de mediación. El análisis de mediación se llevó a cabo siguiendo las recomendaciones de Judd, Kenny y McClelland (2001) para estimar mediación y moderación en diseños intrasujetos. Estos autores recomiendan llevar a cabo el análisis mediante modelos de regresión en donde el criterio son las diferencias en las puntuaciones de la variable dependiente (intención de reclutamiento en nuestro caso) y los predictores son las puntuaciones sumadas de las condiciones intrasujetos, así como las diferencias en las puntuaciones de las mismas (calidez y habilidad percibida, en nuestro caso). Se siguió la recomendación de centrar solamente las puntuaciones sumadas (restándole a cada puntuación bruta la media del grupo) ${ }^{2}$

Debido a que los efectos del sexo y el atractivo de los estímulos sobre la intención de reclutamiento ya fueron estimados en los análisis de varianza anteriormente descritos, el análisis de mediación se concentró en estimar si las diferencias a favor de los estímulos masculinos y atractivos en calidez y habilidad son predictores significativos de las diferencias (también a favor de los estímulos masculinos y atractivos) en la intención de reclutarlos y por tanto representan mediadores significativos del efecto del atractivo y el sexo de las fotografías sobre la intención de reclutamiento.

De acuerdo con Judd et al. (2001), se pueden obtener evidencias a favor de un efecto de moderación si las puntuaciones sumadas de las condiciones intrasujetos representan predictores significativos de las diferencias en la variable dependiente. Por otra parte, se pueden obtener evidencias de mediación si las puntuaciones diferenciales en las condiciones intrasujetos resultan ser predictores significativos de las diferencias en la variable dependiente. Finalmente, el intercepto en estos modelos representa el efecto predictivo de las condiciones más allá de lo que predicen las diferencias entre 
las condiciones, por lo tanto, si este no resulta ser significativamente diferente de cero, se puede inferir mediación total.

En todos los modelos se utilizó la diferencia en la intención de reclutamiento como variable dependiente y como predictores las puntuaciones sumadas y las puntuaciones diferenciales en calidez y habilidad. Las diferencias se calcularon de tal manera que las positivas fueron a favor del reclutamiento, calidez y habilidad de los estímulos masculinos y de los estímulos atractivos, respectivamente. Los resultados de estos análisis se presentan en la Tabla 2.

TABLA 2

Resultados de los análisis de mediación

\begin{tabular}{|c|c|c|c|c|}
\hline \multirow[b]{2}{*}{ Predictores } & \multicolumn{4}{|c|}{$\begin{array}{l}\text { Diferencias en la intención de } \\
\text { reclutamiento a favor de los hombres }\end{array}$} \\
\hline & $B$ & $E E$ & B & \\
\hline Intercepto & 0.30 & 0.08 & & \\
\hline Diferencias en calidez a favor de los hombres & 0.41 & 0.12 & 0.26 & $3.29 * *$ \\
\hline Suna de calidez de hombres y mujeres & 0.03 & 0.03 & 0.08 & 0.99 \\
\hline Intercepto & 0.19 & 0.06 & - & $2.95 * *$ \\
\hline Diferencias en habilidad a favor de los hombr & 0.76 & 0.09 & 0.55 & $8.08^{* * * *}$ \\
\hline \multirow{3}{*}{$\begin{array}{l}\text { Diterencesas en haballdad a a avor de los homb } \\
\text { Suma de habilidad de hombres y mujeres }\end{array}$} & 0.02 & 0.02 & 0.05 & \\
\hline & \multicolumn{4}{|c|}{$\begin{array}{l}\text { Diferencias en la intención de } \\
\text { reclutamiento a favor de los estimulos } \\
\text { más atractivos }\end{array}$} \\
\hline & $B$ & $E E$ & $\mathrm{~B}$ & \\
\hline Intercepto & 0.32 & 0.16 & & $2.03^{*}$ \\
\hline Diferencias en calidez a & 0.67 & 0.09 & 0.54 & $7.70^{* * * *}$ \\
\hline Suma de & 0.07 & 0.04 & 0.11 & 1.60 \\
\hline Intercepto to & 0.54 & 0.10 & & $5.11^{* * * *}$ \\
\hline Diferencias en habilidad a favor de los estímulos más atrac & 0.97 & 0.09 & 0.68 & $11.34^{* * * k}$ \\
\hline Suma de habilidad de los estimulos atractivos y menos atractivos & 0.02 & 0.03 & 004 & 0.66 \\
\hline
\end{tabular}

Nota: $\mathrm{B}=$ peso de regresión, $\mathrm{EE}=$ error estándar, $\beta=$ peso de regresión estandarizado, $t=$ Estadístico de la prueba de significancia,

$* p<0.05 ; * * p<0.01 ; * * p<0.001$.

Fuente: elaboración propia

En los primeros dos modelos de regresión los resultados revelaron que las diferencias en calidez $(\beta=0.26, p=0.001)$ y habilidad $(\beta=0.55, p<$ 0.001 ) de los estímulos masculinos vs. femeninos predijeron significativamente la diferencia en la intención de reclutamiento a favor de los estímulos masculinos, pero la suma de la calidez $(\beta=0.08, p=0.32)$ y habilidad $(\beta=0.05, p$ $=0.47)$ no. De igual manera, el tercer y cuarto modelo de regresión indicaron que las diferencias en calidez $(\beta=0.54, p<0.001)$ y habilidad $(\beta$ $=0.68, p<0.001)$ de los estímulos atractivos vs. no atractivos presentaron efectos significativos en la intención de reclutamiento a favor de los estímulos atractivos; mientras que las sumas de la calidez $(\beta=0.11, p=0.11)$ y habilidad $(\beta$ $=0.04, p=0.51)$ no. En todos los modelos los interceptos resultaron significativos (todas las $p$ $<0.05$ ), indicando mediación parcial.

\section{Discusión}

La relación entre el sexo y el atractivo con variables ocupacionales no siempre ha sido demostrada de manera directa e inequívoca. Ser atractivo no siempre lleva a resultados ventajosos. Algunos estudios han encontrado que las mujeres atractivas pueden ser juzgadas como menos hábiles que las mujeres no atractivas $\mathrm{y}$ por tanto tener menos oportunidades de ser contratadas (Agthea, Spörrlea, \& Manerb, 2010). Otras investigaciones han mostrado que el atractivo físico es ventajoso para los hombres tanto en puestos gerenciales, como en puestos no gerenciales, pero que el atractivo es una ventaja para las mujeres solo en puestos no gerenciales y en muchos casos una desventaja en general (Marlowe, Schneider, \& Nelson, 1996). En otras palabras, la investigación muestra que la relación entre sexo, atractivo, atribuciones y contratación es muy compleja.

El presente trabajo, tuvo como objetivo hacer un aporte al estudio de esta compleja relación mediante un diseño experimental con reclutadores y reclutadoras profesionales. En concordancia con resultados previos, los hallazgos de la presente investigación apoyan la idea de que el atractivo físico y el sexo de los postulantes afectan la intención de reclutamiento y que dicha intención diferencial se encuentra mediada parcialmente por la calidez y habilidad percibida de los estímulos. En otras palabras, los datos sugieren que las personas reclutadoras hacen inferencias sobre las características de los candidatos no solo sobre la base de la información de los CVs, sino también, a partir de las características físicas detectadas a través de la fotografía, y que estas inferencias afectan el proceso de selección de personal.

$\mathrm{Al}$ igual que en estudios previos, el atractivo presentó los efectos más fuertes proporcionando evidencias a favor de la activación del estereotipo de "lo bello es bueno" (Ndobo, 2014; Desrumaux et al., 2009; Farley et al., 1998; Pansu \& Dubois, 2002; Sanhueza et al., 2007; Senior et al., 2007; Sui \& Hong, 2009). Algunos autores han sugerido que la relación entre atractivo físico e intención de reclutamiento recae precisamente 
en las atribuciones de habilidad que se hacen a favor de los estímulos atractivos (Desrumaux et al., 2009), pero pocos son los estudios que han estimado directamente el efecto mediador de estas percepciones.

Se proporciona evidencia de que el atractivo físico aumenta las percepciones de competencia y estas a su vez aumentan las posibilidades de contratación; pero además muestran que el atractivo no solo aumenta las atribuciones de habilidad, sino también las atribuciones de calidez. Una posible explicación para esta generalización de atributos positivos es el conocido efecto de halo (Nisbett \& Wilson, 1977), que ocurre cuando la atribución de una característica positiva a una persona lleva a pensar que todas las características de esa persona son positivas. Otro aspecto destacable de estos resultados es haber detectado el efecto del atractivo en un puesto que no requiere interacción cara a cara con el público, lo que hubiera explicado la preferencia por estímulos atractivos (Pansu \& Dubois, 2002). El "Agente de Call Center" atiende por teléfono y aun así el atractivo privó en la selección de los aspirantes.

El sexo del aplicante presentó también un efecto significativo, pero de menor tamaño y restringido (moderado) por el sexo de la persona participante, siendo las reclutadoras particularmente severas con los estímulos femeninos poco atractivos. En estudios previos, el sesgo en contra de las mujeres se ha observado principalmente en puestos que se consideraban típicamente masculinos. En este estudio se trabajó con un puesto sexualmente neutro, proporcionando evidencia de que ante condiciones equivalentes, el hombre tiende a presentar ventajas. En otras palabras, cuando los criterios objetivos en la evaluación de los CVs son equiparables, los aspectos subjetivos en la valoración de los aplicantes emergen, y es aquí en dónde aparecen con mayor fuerza los estereotipos asociados al sexo, y es aquí en dónde la mujer se encuentra más severamente evaluada. Estos resultados dan cuenta de la persistencia de creencias culturales sobre el rol dominante asignado a los hombres, considerados más fuertes, capaces y competentes (Gorman, 2005).
El efecto moderador del sexo de la persona reclutadora en el proceso de percepción y decisión sobre los estímulos resulta intrigante. La mayoría de los estudios aquí revisados no encontraron diferencias significativas según el sexo del reclutador en los procesos de percepción y evaluación en los contextos laborales. En el presente estudio, en cambio, los reclutadores tendieron a diferenciar a favor de los estímulos atractivos sin distingo de su sexo, mientras que las reclutadoras tendieron a castigar más a los estímulos femeninos, sobre todo cuando no eran atractivos. A diferencia de los resultados de los estudios sobre rivalidad intrasexual en contextos laborales, en donde se ha observado que las personas reclutadoras tienden a presentar sesgos ante potenciales rivales atractivos del mismo sexo (Agthea et al., 2010), las mujeres reclutadoras en este estudio tendieron a ser más severas con los estímulos de mujeres menos atractivas. Adelantar una explicación a este efecto sería muy arriesgado. Debido al reducido número de reclutadores y dado el reducido tamaño del efecto de interacción (menos del $4 \%$ de varianza explicada), lo más recomendable sería llevar a cabo una replicación del presente estudio con el fin de estimar la consistencia de este inusual resultado.

\section{Limitaciones}

El presente estudio no está exento de limitaciones. Una de ellas es que la información proporcionada en los CVs era ficticia y aunque fue evaluada en los estudios piloto, pudo haber estado muy distanciada de las características de los CVs que se evalúan en el proceso de reclutamiento en contextos reales. Las fotografías utilizadas en el estudio pudieron también haber presentado aspectos idiosincráticos que pudieron afectar las reacciones. A pesar del extenso pilotaje de las mismas, las fotografías podrían haber variado no solo en la dimensión de atractivo, sino en otras dimensiones no consideradas. Futuros estudios podrían beneficiarse de una mayor estandarización de los estímulos fotográficos mediante modificaciones 
computarizadas de caras ampliamente validadas. Finalmente, el contexto de aplicación de los cuestionarios no fue totalmente controlado, ya que se aplicaron los protocolos en diferentes momentos y lugares, lo que pudo incidir en las respuestas.

\section{Conclusión}

A pesar de estas limitaciones, los resultados del presente estudio apoyan la idea de que los estereotipos influyen en la percepción, la evaluación, los afectos y los comportamientos humanos, no solo en contextos intergrupales sino también en contextos interpersonales y laborales. En el proceso de selección de personal la presencia de aspectos subjetivos (como los estereotipos que se activan ante el sexo y el atractivo físico de los postulantes) parece ser inevitable. Los y las profesionales a cargo del proceso de selección de personal no necesariamente están conscientes de esos sesgos y seguramente realizan su trabajo de la manera más profesional, tratando de ser lo más objetivos posible, por lo que se verían muy beneficiados al implementar estrategias para evitar el innegable efecto que los estereotipos pueden producir en sus decisiones.

\section{Agradecimientos}

La investigación contó con financiamiento del Fondo de Apoyo para Trabajos Finales de Graduación de la Vicerrectoria de Investigación de la Universidad de Costa Rica, otorgado a la primera autora.

Las autoras agradecen a Natalia Sánchez y Margie Blanco, por su asesoría a lo largo del desarrollo del proyecto de investigación.

\section{Referencias}

Agthea, M., Spörrlea, M., \& Manerb, J.K. (2010). Don't hate me because I'm beautiful: Anti-attractiveness bias in organizational evaluation and decision making. Journal of experimental Social Psychology, 46(6), 11511154. http://dx.doi.org/10.1016/j.jesp.2010 .05 .007

Bauer, C., \& Baltes, B. (2002). Reducing the effects of gender stereotypes on performance evaluations. Sex Roles, 47(9-10), 465-476. Recuperado de http://www.cos.gatech.edu/facultyres/Di versity_Studies/BauerBaltes.pdf

Caprariello, P., Cuddy, A., \& Fiske, S. (2009). Social Structure Shapes Cultural Stereotypes and Emotions: A causal test of the stereotype content model. Group Processes y Intergroup Relations, 12(2), 147-155. http:// dx.doi.org/10.1177/1368430208101053 .

Caro, F., García, M., Rodríguez, C., \& Jiménez, G. (2007). Percepción de las habilidades y aceptación de las mujeres directivas en las empresas de comunicación. Zer: Revista de Estudios de Comunicación, 22, 253-266. Recuperado de http://www.ehu.eus/zer/he meroteca/pdfs/zer22-13-caro.pdf

Cuddy, A. J. C., Fiske, S. T., Kwan, V. S. Y., Glick, P., Demoulin, S., Leyens, J.P., ... Ziegler, R. (2009). Stereotype Content Model across cultures: Universal similarities and some differences. British Journal of Social Psychology, 48(1), 1-33. http://dx.doi.org/10 .1348/014466608X314935

Desrumaux, P., De Bosscher, S., \& Léoni, V. (2009). Effects of facial attractiveness, gender, and competence of applicants on job recruitment. Swiss Journal of Psychology, 68(1), 33-42. http://dx.doi.org/10.1024/14 21-0185.68.1.33

Eagly, A. H., Ashmore, R. D., Makhijani, M. G., \& Longo, L. C. (1991). What is beautiful is good, but...: A meta-analytic review of the physical attractiveness stereotype. Psychological Bulletin, 110(1), 109-128. Recuperado de https://www.uni-muenster. de/imperia/md/content/psyifp/aeechterhoff /wintersemester2011-12/vorlesungkommp erskonflikt/eaglyetal_metaaphysattracstere o psychbull1991.pdf

Farley, S., Chia, R., \& Allred, L. (1998). Stereotypes about attractiveness: 
when beautiful is not better. Journal of Social Behavior and Personality, 13(3), 479-492. Recuperado de https:// www.researchgate.net/publication/297492 615_Stereotypes_about_attractiveness_W hen_beautiful_is_not_better

Fiske, S., Cuddy, A., Glick, P., \& Xu, J. (2002). A model of (often mixed) stereotype content: Competence and Warmth respectively follow from perceived status and competition. Journal of Personality and Social Psychology, 82(6), 878-902. http://dx.doi.org/1037//0022-351 4.82.6.878

Fiske, S., \& Taylor, S. (2013). Social cognition: from brain to culture. (Second Edition). New York: MacGraw-Hill.

Glick, P., Zion, C., \& Nelson, C. (1988). What Mediates Sex Discrimination in Hiring Decisions? Journal of Personality and Social Psychology, 55(2), 178-186. http://dx.doi.or $\mathrm{g} / 10.1037 / 0022-3514.55 .2 .178$

Godoy, L., \& Mladninic, A. (2009). Estereotipos y roles de género en la evaluación laboral y personal de hombres y mujeres en cargos de dirección. Pykhe, 18(2), 55-64. Recuperado de http://www.scielo.cl/pdf/psykhe/v18n2/ art04.pdf

Gorman, E. (2005). Gender Stereotypes, SameGender Preferences, and Organizational Variation in the Hiring of Woman: Evidence from Law Firms. American Sociological Review. 70, 702-728. Recuperado de https://sociology.virginia.ed u/sites/sociology.virginia.edu/files/GormanGenderStereotypesSameGender.pdf

Honekopp, J., Bartholomé, T., \& Jansen, G. (2004). Facial attractiveness, symmetry and physical fitness in young women. Human Nature, 15(2), 147-167. http:// dx.doi.org/10.1007/s12110-004-1018-4.

Horcajo, J., Briñol, P., \& Becerra, A. (2009). Los efectos de la activación de estereotipos sobre la evaluación de candidatos en un contexto experimental de Selección de Personal. Revista Latinoamericana de Psicología, 41(2), 349-359. Recuperado de http://www.redalyc.org/pdf/805/805114 96012.pdf
Judd, C.M., Kenny, D. A., \& McClelland, G.H. (2001). Estimating and Testing Mediation and Moderation in WithinSubject Designs. Psychological Methods, 6(2), 115-1134. http://dx.doi.org/10.1037/ 1082-989X.6.2.115

Keppel, G., \& Wickens, T. (2004), Design and Analysis: A Researcher's Handbook, 4th edition, Prentice Hall.

Levin, I., Rouwenhorst, R., \& Trisko, H. (2005). Separating gender biases in screening and selecting candidates for hiring and firing. Social Behavior y Personality: An International Journal, 33(8), 793-804. http://dx.doi.org/1 0.2224/sbp.2005.33.8.793 .

Marlowe, C. M., Schneider, S. L., \& Nelson, C. E. (1996). Gender and attractiveness biases in hiring decisions: are more experienced managers less biased? Journal of Applied Psychology, 81, 11-21. Recuperado de http:// scholarcommons.usf.edu/psy_facpub/1890/

$\mathrm{Ng}$, E. \& Wiesner, W. (2007). Are Men Always Picked Over Women? The Effects of Employment Equity Directives on Selection Decisions. Journal of Business Ethics, 76(2), 177-187. Recuperado de http://www.jstor. org/stable/25075506

Ndobo, A. (2014). Attractiveness effect and the hidden discourse of discrimination in recruitment: the moderating role of job types and gender of applicants. Revue internationale de psychologie sociale, 27(1), 127-144. Recuperado de https://www.cairn.info/load_pdf.php?ID ARTICLE $=$ RIPSO_271_0127

Nisbett, R. E., \& Wilson, T. D. (1977). Telling more than we can know: Verbal reports on mental processes. Psychological Review, 84(3), 231-259. Recuperado de http://peop le.virginia.edu/ $\sim \mathrm{tdw} /$ nisbett\&wilson.pdf

Oficina Internacional del Trabajo [OIT] (2007). La igualdad en el trabajo: afrontar los retos que se plantean. Conferencia Internacional del trabajo. Reunión 96. Ginebra: OIT.

Pansu, P., \& Dubois, M. (2002). The effects of face attractiveness on pre selective recruitment. Swiss Journal of Psychology, 
61(1), 15-20. http://dx.doi.org/10.1024//14 21-0185.61.1.15

Patzer, G. (2006). The power and paradox of physical attractiveness. Florida: BrownWalter.

Penton-Voak, I., Jones, B., Little, A., Backer, S., Tiddeman, B., Burt, D., \& Perrett, D. (2001). Symmetry, sexual dimorphism in facial proportions and male facial attractiveness. The Royal Society, 268, 1617-1623. http://dx.doi.org/10.1098/ rspb.2001.1703

Przygodzki-Lionet, N., Olivier, J., \& Desrumaux, P. (2010). The effects of facial attractiveness, sex, internality of applicants on hiring decisions for managerial and nonmanagerial jobs. Studia Psychologica, 52 (1), 53-57. Recuperado de

Reuben, E., Sapienza, P., \& Zingales, L. (2014). How stereotypes impair women's careers in science. Proceedings Of The National Academy Of Sciences Of The United States Of America, 111 (12), 4403-4408. http://dx .doi.org/10.1073/pnas.1314788111

Rice, L., \& Barth, J. (2016). Hiring Decisions: The Effect of Evaluator Gender and Gender Stereotype Characteristics on the Evaluation of Job Applicants. Gender Issues, 33(1), 1-21. http://dx.doi.org/10.1007/s121 47-015-9143-4 .

Rhodes, G. (2005). The evolutionary psychology of facial beauty. Annual Review Psychology, 57, 199-226. http://dx.doi.org/10.1146/ann urev.psych.57.102904.190208

Ruvalcaba-Coyaso, FJ., \& Vermonden, A. (2015). Lógica difusa para la toma de decisiones y la selección de personal. Universidad y Empresa, 17(29), 239-256. http://dx.doi.org/10.12804/rev.un iv.empresa.29.2015.10

Sanhueza, C., Bravo, R., \& Giusti, O. (2007). La belleza y su efecto en el Mercado Laboral: Evidencia para Chile. (Documento de Trabajo $\mathrm{N}^{\circ}$ 204). Departamento de Economía. ILADES -Georgetown University.

Senior, C., Thomson, K., Badger, J., \& Butler, M. (2007). Interviewing strategies in the face of beauty: a psychophysiological investigation into the job negotiation process. Annals of The New York Academy Of Sciences, 1118(1), 142-162. http:// dx.doi.org/10.1196/annals.1412.005

Shannon, M., \& Stark, P. (2003). The influence of physical appearance on personnel selection. Social Behavior and Personality, 31 (6), 613-624. http://dx.doi.org/10.2224/s bp.2003.31.6.613

Smith-Castro, V., \& Perez, R. (2007). Atribuciones estereotípicas de calidez y habilidad en jóvenes universitarios. Revista de Ciencias Sociales, 117-118, 129-147. Recuperado de http://revistas.ucr.ac.cr/index.php/social es/article/viewFile/11019/10399

Sui, J., \& Hong C. (2009). Can beauty be ignored? Effects of facial attractiveness on covert attention. Psychonomic Bulletin y Review, 16(2), 276-281. http://dx.doi.org/1 0.3758/PBR.16.2.276

Tooby, J., \& Cosmides, L. (2005). Conceptual foundations of evolutionary psychology. In D. M. Buss (Ed.), The Handbook of Evolutionary Psychology (pp. 5-67). Hoboken, NJ: Wiley.

Viswesvaran, C., Schmidt, F.L., \& Ones, D.S. (2005). Is There a General Factor in Ratings of Job Performance? A MetaAnalytic Framework for Disentangling Substantive and Error Influences. Journal of Applied Psychology, 90(1), 108-131. http://d x.doi.org/10.1037/0021-9010.90.1.108

\section{Notas}

* Artículo de investigación.

1 Los diagnósticos de normalidad y homogeneidad de las varianzas fueron satisfactorios. No se presentan aquí por razones de espacio pero pueden ser consultados a la primera autora. Es importante notar que previamente a los análisis principales se realizaron análisis de covarianza $2 \times 2 \times 2$ incluyendo los años de experiencia de las personas reclutadoras como covariable. No se encontraron efectos 
significativos de la covariable o de las interacciones en las que se vio involucrada (todas las $\mathrm{Fs}_{1}, 149<0.19$, todas las ps $>$ 0.66), por lo que aquí solo se presentan los resultados de los análisis previstos originalmente.

2 Debido a que el número de reclutadores masculinos era muy reducido, realizamos el análisis para toda la muestra. Recuérdese que el sexo de la persona reclutadora no afectó la dirección de las relaciones entre sexo y atractivo del estímulo e intención de reclutamiento, solo su magnitud. 